ecchymosen bei Neugeborenen, habe ich durch Herrn Dr. Reuss sehr werthvolle Beiträge erhalten.

In 165 Sectionen kamen dieselben 41 Mal zur Beobachtung und zwar in folgender Vertheilung am gesammten Klappenapparate: 13 Mal in der Trieuspidalis; $8 \mathrm{Mal}$ in der Mitralis; $17 \mathrm{Mal}$ in der Tricuspidalis und Mitralis zugleich; 2 der Mal in Tricuspidalis, Mitralis und den Semilunarklappen der Art. pulmonalis zugleich; 1. Mal in der Mitralis und den Klappen der Art. pulm. zugleich.

Jene 165 Sectionen betrafen 128 lebendig und 37 todt geborene Kinder. Unter den lebend Geborenen zeigten 31, unter den todt Geborenen 10 dieselben Ecchymosen im Gewebe der Klappen des Herzens.

Nach einem von E'sässer gegebenen Berichte über die Ereignisse in der Gebäranstalt des Catharinen-Hospitals im Jahre 1844 (vgl. Medic. Correspondenzblatt Bd. XIV. S. 297.) wurden Blutextravasate im Gewebe der Mitralis und Tricuspidalis in mehr als der Hälfte der Sectionen vorgefunden - bei Kindern von der Geburt bis zum 23sten Tage.

Indem ich die Betrachtung der etwa bedingenden Momente, sowie des Einflusses dieser Blutergússe auf spätere Klappenveränderungen einer anderen Gelegenheit vorbehalte, genügt es mir einstweilen in weiteren Kroisen dic Aufmerksamkeit auf diesen Gegenstand gelenkt zu haben.

\title{
XII.
}

\section{Zur Theorie der Percussion.}

Von Dr. C. Schweigger, Privat-Docent zu Halle.

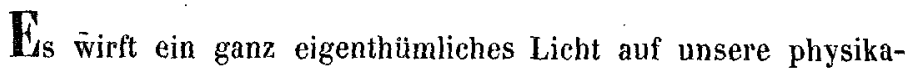
lische Diagnostik, dass dieselbe von je her eine Menge von Erscheinungen zu benutzen wusste, ohne uber die physikalischen 
Ursachen derselben recht im Klaren zu sein. Was bei der Auscultation der Lungen überhaupt zu hören jst, das hatte schon Laennec so vollständig wahrgenommen, dass seine Nachfolger das empirische Material nur um sehr weniges vermehren konnten; und das, was er hörte, wusste er so richtig zu verwerthen, dass seit dem die Fortschritte in der Diagnostik der Lungenkrankheiten mit dem ersten Sehritte, den Laennec selbst gethan hat, nicht zu vergleichen sind. Der wissenschaftliche Fortschritt bestand seit Laennec mehr in der physikalischen Begründung der auscultatorischen Zeichen, als in einer Erweiterung und Vermehrung dieser Zeichen selbst. Ganz ähnlich verhält es sich mit der Percussion. Die allseitige Anwendung derselben hat seit langer Zeit nur sehr wenige neue Resultate geliefert, man hat die empirisch bekannten Zeichen in praxi richtig zu verwerthen gewusst, während die wissenschaftliche Discussion_sich. noch mit den Cardinalfragen der Theorie beschäftigte. Wo ist die eigentliche Quelle des Schalles, den wir beì der Percussion hören? Vom theoretisehen Standpunkte aus sollte man meinen, eine zuverlässige praktische Anwendung der Percussion sei nicht möglich, so lange diese Frage nicht vollständig entschieden ist. Angesichts der Thatsache, dass dies nicht der Fall ist, dass man die Resultate der Percussion zum grössten Theile richtig verwerthen kann, wenn man auch die Frage, wo der Percussionsschall eigentlich entsteht, unentschieden lässt, kömnte man auf den Gedanken kommen, dass derartige Erörterungen den Bedürfnissen der Praxis überhaupt nicht entsprächen, und da schliesslich doch alles auf die praktische Verwertbung ankommt, so witrden wir uns auf die Discussion der angeregten Frage nicht einlassen, wenn wir nicht hoffen dürften, cinen Gesichtspunkt zu gewinnen, der die Entscheidung dieser Frage auch für die praktische Ausübung der Percussion wichtig macht.

Betrachten wir zunächst die verschiedenen Ansichten über die Entstehung des Percussionsschalles, so lässt Piorry seinen Lungenton in der in den Lungen enthaltenen Luft entstehen. Stokes dehnte diese Ansicht sogar so weit aus, dass er eine Abnahme des Tones bei Anfüllung der Bronchien mit Secreten und bei disseminirten kleinen 'luberkeln annahm. Auch nach 
Williams*) entsteht der Percussionsschall ,durch die elastische Luft, welche sich im Innern in dem schwammigen Gewebe der lungen befindet und den Brustwandungen freie Schwingungen gestattet. Aber damit die Schwingung hervorgebracht werde, müssen die Wände einen gewissen Grad elastischer Spannung besitzen. Denn, sind dieselben schlaff und geben bei der Percussion nach, so wird man keinen Ton hören ausser dem leisen und dumplen Tone, der durch den Anschlag des Finger's auf die Oberfläche entsteht. Der naturliche feste Bau der Brust, der knöcherne Kasten mit seinen elastischen Bändern und Knorpeln, und die mehr oder weniger dichten Muskeln und Integumente, mit welchen sie bedeckt ist, sind im Allgemeinen wohl dazu geeignet, die Einwirkung einer äusseren Percussion zum Innern zu leiten. Aber, hat sich die Elasticität der Knorpel nur in etwas verloren, oder sind die Integumente aufgedunsen durch Oedem, Fett oder andere Ursachen, so wird der Wiederhall der Percussion verhältnissmässig geschwäcbt, und hier muss man wohl diese Veränderungen des Brusttones von denjenigen Veränderungen unterscheiden, welche von dem Zustande der inneren organe bedingt werden. $\mathrm{Da}$ in dem natürlichen und gesunden Zustande die Helligkeit und Stärke des Brusttons von der Anfüllung der Lungen mit Luft, sowie von der Dünnheit und der Spannung der Brustwandungen abhängt, so ist es klar, dass diejenigen Theile der Brust am besten tönen werden, welche am meisten diese Bedingungen erfillen." Man sieht, dass Williams Ansichten über die Betheiligung der Thoraxwandungen beim Percussionsschalle der Brust in vielen Punkten mit denen Skoda's zusammenfallen, welche ich als allgemein bekannt voraussetzen dart **).

In einer ausführlichen Abhandlung über die Betheiligung der

*) Charles Williams, Pathologie und Diagaose der Krankheit der Brust, ïbers. von Dr. Herm. Velten. Bom 1838. S. 22.

**) Skoda (5. Autl. S.5.) schreibt Williams eine viel entschiedenere Ansicht uiber die Betheiligung der Brustwandungen am Percussionssclalle zu, als sich aus der hier citirten Stelle erseluen lässt. Vielleicht erklärt sich dieser Widerspruch daraus, dass Williams seine Ans̀ichten später geändert hat; wenigstens scheint Skoda eine spätere Ausgabe (Leiprig 1841; cf. Skoda S. 12) benutzt zu baben, welcie mir nicht zugänglich war. 
Brustwandungen am Percussionsschalle spricht sich Mazonn*) dahin aus, dass der Schall zunächst durch die Schwingungen der Brustwandungen entstehe und in der in den Lungen enthaltenen Luft consonire und verstärkt werde.

Nach Hoppe**) entsteht der Percussionsschall der Brust lediglich durch die Schwingungen der Brustwandungen; die Menge der im Thorax enthaltenen Luft übt keinen Einfluss auf den Percussionsschall der Wandungen aus; luftleere Massen (Lungeninfiltrate, pleuritische Exsudate u. s. w.), welche der Thoraxwand anliegen, wirken auf dieselbe wie ein Dämpfer auf die Schwingungen von Saiten. Der Pereussionssehall des Thorax ist nicht tympanitisch, weil das gespannte Lungengewebe den Schwingungen der Wandungen ein bedeutendes Hinderniss in den Weg setzt; der Schall wird in Folge dessen tympanitisch, sobald die Elasticität der Lunge ausser Wirkung tritt, $z$. B. bei der sogenannten Compression der Lunge durch pleuritisches Exsudat und bei Exsudationen in das Gewebe der Lunge, so lange als dieselbe noch nicht luftleer ist.

Wintrich***) sucht die Quelle des tympanitisehen Percussionsschalls einer aus der Brusthöhle herausgenommenen und einfach extrahirten Lunge im elastischen Lungengewebe selbst; dasselbe soll durch die Percussionserschütterung in Schwingungen versetzt werden, ,welche tonherrschend die enthaltene Luft nur zur Schallverstärkung zwingen." Consequenter Weise leitet er dann auch den nicht tympanitischen Percussionsschall der Lunge im geschlossenen Thorax ebenfalls von den Schwingungen des elastischen Lungengewebes ab.

Es wären sonach die Medien, in denen der Percussionsschall der Brust möglicher Weise entstehen kann, vollstảndig erschöpft. Wir haben nach einander gesehen, dass man denselben von der in den Lungen enthaltenen Luft, von den Schwingungen der Thoraxwandungen und endlich von den Schwingungen des elastischen Lungengewebes selbst ableitete. Eine neue Ansicht aufzustellen,

*) Mazonn, Prager Vierteljabrssclirift Bd. 36 .

**) Virchow's Archiv Bd. 6. Heft 2. S. 166. 168. 170.

***) Virchow, Handb. A. Pathol. Ba. 5. S. 18. 25. 
dürfte unmöglich sein, jedenfalls aber müssen wir uns aufgefordert finden, diese verschiedenen Ansichten experimentell zu prüfen.

\section{I.}

Der tympanitische Percussionsschall.

Wintrich stützt seine eben mitgetheilte Ansicht durch folgende Deduction. Eine von glatten, schallreflexionsfähigen Wänden eingeschlossene Luftsäule giebt bei der mittelbaren Percussion einen tympanitischen Schall. Die Höhe und Tiefe dieses Schalles ist abbängig vom längsten Durchmesser des percutirten Luftraumes und von der Grösse der freien Mündung dieses Raumes, ganz analog den bekannten physikalischen Gesetzen offener und geschlossener Pfeifen. So giebt cine in einem oben und unten offenen Cylinder eingeschlossene Luftsäule bei der mittelbaren Percussion einen höheren Ton, als wenn die Bodeuwand des Cylinders geschlossen wird. Der Ton wird noch tiefer, wenn auch die Oeffnung, oberhalb deren man percutirt, verengert wird. Abweichend von diesen physikalischen Gesetzen findet Wintrich das Verhalten einfach retrahirter Lungen bei der Percussion. Der Percussionsschall der Lunge ist nach Wintrich zu tief, als dass er, in den relaxirten Bläschen als Schallräumen” entstehen könnte. „Schon eine Luftsäule von 6 Linien Höhe giebt einen tympanitischen Schall, dessen Höhe unendlich differirt von der Tiefe des tympanitischen Schalles einer contrahirten Lunge, sei diese selbst die kleine Lunge eines Kaninchens. Auch findet sich bei näherer Untersuchung, z. B. an Kälberlungen u. s. w., dass die kleineren Lappen einen höheren tympanitischen Schall geben als die grösseren, während doch die Bläschen in den kleineren Lappen nicht kleiner als in den grösseren sind *)."

Wintrich kommt daher zu dem Resultat: die Meinung, dass die Lufträume der relaxirten Lungenbläschen einen unterscheidbar hohen tympanitischen Percussionsschall zu geben vermögen, müsse als eine untaugliche und unstatthafte betrachtet werden. Aber nicht blos die Lungenbläschen, argumentirt $W$ intrich weiter, son-

*) I. c. S. 17 .

Archiv f. pathol. Anat. Bd, XI. Hft. 2. 
dern auch die Bronchien enthalten luftsäulen; entsteht der tyrapanitische Ton in diesen? Auch diese Frage glaubt Wintrich verneinend beantworten zu müssen, denn die Höhe des tympanitischen Schalles bleibt sich auch dann noch gleich, wenn man entweder die Trachea oder die freien Oeffnungen der einzelnen Bronchien auf der Schnittä̈che eines abgetrennten Lungenstuickes durch eine dünne. Fleischplatte oder eine feuchte Blase schliesst. Analog den physikalischen Gesetzen offener und gedeckter Pfeifen müsste nämlich der Percussionsschall tiefer werden: „also können unmöglich die in den Bronchien eingeschlossenen Luftsäulen die Höhe und Tiefe des tympanitischen Schalles bestimmen *)."

In der Luft der Lunge kann also nach Wintrich der Percussionsschall der Lunge überhaupt gar nicht entstehen; als einzig mögliche Quelle desselben bleibt dann nur noch das elastische Gewebe derselben übrig. Dass ein grösserer Lungenflügel tiefer schallt als ein kleinerer derselben Lunge, ist nach Wintrich ganz einfach darin begründet, dass je nach der Masse und Grösse einer schwingenden Membran u. s. w. bei gleicher Spannung der Ton immer eine verschiedene Höhe zeigt. Ganz übereinstimmend mit seinen Ansichten findet es Wintrich, dass der tympanitische Schall der Lunge höher wird, wenn man die Spannung des Lungengewebes durch Dehnung vermehrt. „Somit hängt die Höhe des tympanitischen Schalles contrahirter Lungen von der Spannung des Lungengewebes ab und die enthaltene Luft wirkt nur wie ein Resonanzboden - schallverstärkend." Wie es möglich sei, dass die in den Lungenzellen entbaltenen Lufträume zu klein sein sollen, um selbst tönend irgend einen wahrnehmbaren Schall zu geben, aber gross genug, um vermöge mitgetheilter Schwingungen den Schall $\mathrm{zu}$ verstärken, wird nicht weiter in Betracht gezogen.

Bei der von Wintrich vorgebrachten Thatsache, dass der tympanitische Percussionsschall durch Anspannung des Lungengewebes höher wird, muss sich Jedem sofort die Vermuthung aufdrăngen, diese Erscheinung sei einfach darin begründet, dass mit der Dehnung eines Lungenlappens die Dicke (d. i. Höhe) desselben abnehme, und von der verminderten Höhe der percutirten Luft") 1. c. S. 19. 


\section{5}

säule, nicht von der Spannung des Gewebes hänge die Erhöhury des tympanitischen Schalles ab. In der That hat auch Dr. M. A. Körner*) die eben ausgesprochenen Bedenken erhoben, ohne aber darauf Rücksicht zu nehmen, dass Wintrich schon selbst, wenn auch auf eine etwas unzulängliche Weise, diesem Einwurfe zuvorzukommen gesucht hat.

Nimmt man nämlich mit Körner an, die in irgend einem Lungentheile enthaltene Luft schalle wie ein Continuum, so richtet sich die Höhe dieses Schalles nicht nach der Grösse des Durchmessers, in dessen Richtung man percutirt, sondern nach der Grösse des längsten Durchmessers, nnd Wintrich hat dann vollkommen Recht, wenn er sagt, dass man, indem man den längsten Durchmesser der Luftsäule noch verlängert, cher ein Tieferwerden des Schalles erwarten müsse. Aber die in einem Lungentheile eingeschlossene Luft ist eben kein Continuum, sondern ihre Continuität ist durch viele Zwischenkörper, die Wandungen der Lungenbläschen, der Bronchien u. s. w., unterbrochen, und es muss also erst gezeigt werden, in wie weit die Gesetze, welche bei der Percussion von Luftrüumen gelten, sich auf die in den Lungen enthaltene Luft anwenden lassen. So viel ist jedenfalls richtig, dass es nicht allein die Höhe der Luftsäule, resp. die Dicke des percutirten Lungenstückes ist, welche die Höhe des tympanitischen Percussionsschalles bestimmt. So giebt z. B. ein aus einer Lunge Kerausgeschnittenes Stück, welches kleiner ist als das Plessimeter, mittelst welchem man es percutirt, einen höheren tympanitischen Ton als ein eben so dickes aber grösseres Stück. Die Percussionserschütterung, die wir auf irgend einen Lungentheil ausüben, planzt sich wahrscheinlich nicht bloss in der Richtung des Percussionsstosses fort, sondern auch nach allen anderen Richtungen; bis auf welche Entfernung hin diese seitliche Ausbreitung stattfindet, habe ich nicht ermitteln können. Jedenfalls aber glaube ich die Annahme für gerechtfertigt halten zu dürfen, dass die durch die Percussion angeregten tönenden Schwingungen sich am leichtesten in der Richtung der durch die Percussion ausgeübten Compression ausbreiten werden, und dass die Ausbreitung der-

*) Zeitschr. der Gesellscb. der Aerzte za Wien 1855. S. 307. 
selben nach allen anderen Richtungen hin an der Elasticität des Lungengewebes ein verhältnissmässig grösseres Hinderniss findet, welches durch Dehnung und Anspannung der Lunge gewiss nicht vermindert wird. Wintrich scheint mir demnach das Gewicht des Einwurfes, den er sich selbst macht, doch zu gering angeschlagen żu haben. Nehmen wir z. B. an, die seitliche Ausbreitung der durch die Percussion ausgeübten Compression betrage im gedehnten wie im nicht gedehnten Lungengewebe $\frac{1}{2}$ Zoll, so wird es gleichgültig sein, ob das percutirte Lungenstück 4 oder 8 Zoll lang ist. Die Höhe des tympanitischen Percussionstones wird immer von der Dicke des Lungentheiles, resp. von der Höhe der Luftsäule abhängig sein.

Eben so wenig hat aber Wintrich seine Ansicht, dass der tympanitische Percussionsschall durch die Schwingungen des elastischen Lungengewebes entstehe, hinlänglich begrundet. Er spricht nämlich von vorn herein die Ueberzeugung aus, man werde die Annahme wohl kaum verwerfen, dass das elastische Lungengewebe bei vorhandenem Gleichgewichte der umgebenden Luft' in einer solchen Spannung durch sich selbst erhalten sei, dass es bei der Percussion in tönende Schwingungen gerathen könne. Die schlaffe Beschaffenheit, welche eine aus dem Thorax herausgenommene Lunge zeigt, spricht nicht gerade sehr für diese Annahme; man begreift auch gar nicht, wo so eine Spannung herkommen soll, da nichts die Ausgleichung derselben hindert. Man kann sich die elastischen Kräfte einer aus dem Thorax herausgenommenen Lunge nicht anders als concentrisch wirkend denken, und wir sehen, dass die Lunge, indem sie diesem Zuge folgt, eine allseitige Verkleinerung erfährt; auch die in den Lungen enthaltene Luft kann nicht die Ursache der von Wintrich vorausgesetzten Spannung sein, so lange sie sich noch im Gleichgewichte mit der umgebenden Luft befindet. Wäre das Lungengewebe aber wirklich nicht durch sich selbst in einer solchen Spannung erhalten, so müsste diese Spannung nachlassen, wenn man den mechanischen Zusammenhang desselben aufhebt, der Ton müsste dann tiefer werden. Körner*) hat gezeigt, dass dies nicht der Fall ist. „Spaltet man einen ") l. c. S. 311 . 
Lungenlappen in 3, 4, 6 oder noch mehr dünne Schichten, so giebt jede Schicht für sich einen tympanitischen Schall, der um so höher ist, je dünner die percutirte Lungenschicht." „Legt man nun alle durch Spaltung eines Lungenlappens erbaltenen Schichten nach und nach wieder über einander, so wird der tympanitische Schall mit jeder neu aufgelegten Schicht tiefer, und endlich nahezu gerade so tief, wie er an dem noch ungespaltenen Lungenlappen durch Percussion entstanden war." Dieses Experiment lässt sich nur mit einiger Vorsicht verwerthen, denn durch das Uebereinanderlegen der einzelnen Lungenschichten wird natürlich auch die Masse des elastischen Lungengewebes vermehrt.

Wir können aber auch positive Beweise dafür vorbringen, dass sich der Percussionsschall der Lunge in gewissen Beziehungen eben so verhalte, wie der Schall eines begrenzten Luftraumes, indem wir den Schall nach Belieben höher oder tiefer machen, ohne an der elastischen Spannung der Lunge das Geringste zu ändern. Das Experiment ist einfach und unzweideutig. Der Schall eines in freier Luft schwebend gehaltenen Lungenlappens ist höher, als wenn man denselben Lungentheil an derselben Stelle auf einer nicht schallenden Unterlage percutirt. Wir finden demnach im Verhalten des Lungenpercussionsschalles die vollständigste Analogie mit dem Gesetze, dass der Schall eines oben und unten offenen Cylinders tiefer wird, wenn man den Boden desselben durch einen Körper von hinreichender Schallreflexionsfähigkeit verschliesst.

Wintrich hätte gewiss für die Erklärung des Percussionsschalles seine Zuflucht gar nicht zum elastischen Lungengewebe genommen, wenn er nicht von der Ansicht ausgegangen wäre, dass das zwischen den einzelnen Lufträumen befindliche elastische $\mathrm{Ge}-$ webe der Lunge das Zustandekommen regelmässiger Schallwellen in der Luft der Lunge verhindern müsse, er würde sonst nicht die in den Bronchien enthaltenen Luftsäulen von den Lungenbläschen als Schallräumen zu trennen für nöthig gefunden haben.

Man kann sich durch ein einfaches Experiment uberzeugen, dass zum Zustandekommen sogenannter stehender Schallwellen in der Luft es durchaus nicht nöthig ist, dass dieselbe ein Continuum 
bilde. Schneidet man aus einer Lüge mehrere Stücke von einigen Zoll Durchmesser und 4-5 Linien Dicke, so giebt jedes, auf einer nicht schallenden Unterlage percutirt, einen tympanitischen Schall; legt man zwei Stücke über einander und zwischen beide ein grosses Stück feuchte Blase, welches die Schnittlächen reichlich überragt, so bekommt man einen Percussionsschall, der tiefer ist als der jedes einzelnen Stuickes. Der tiefe Schall entsteht hier offenbar durch ein System von Schallwellen, welches beiden Stiicken gemeinschaftlich angehört und dessen Regelmässigkeit nicht einmal durch eine oder mehrere dazwischen liegende Blasenwände vernichtet wird - die zarten Scheidewände der einzelnen Lungenbläschen mögen demuach die Regelmässigkeit dep Schallwellen wohl einigermaassen stören, sie können sie aber nicht vollständig vernichten.

Wir müssen demnach für den tympanitischen Schall einer aus dem Thorax herausgenommenen Lunge die Behauptungen Wintrich's über die Betheiligung des Lungengewebes an der Entstehung des Schalles zurückweisen, und die alte Ansicht, dass der Schall in der Luft der Lunge entstehe, anerkennen.

II.

Der nicht tympanitische Schall.

Es ist eine völlig unmotivirte Aeusserung Skoda's, dass es mit den Gesetzen der Physik in Widerspruch scheine, dass die Lunge bei einem geringeren Luftgehalte einen tympanitischen Schall giebt, während derselbe bei vermehrter Luftmenge nicht tympanitisch ist. Die Regelmässigkeit der Schwingungen hängt nicht ab von der Grösse des schallenden Körpers. Der tympanitische Percussionsschall einer mit Luft mässig gefüllten Blase wird bei ganz gleichem Luftgehalt nicht tympanitisch, wenn man das Plessimeter so stark aufdrückt, dass die enthaltene Luft comprimirt und die Blasenwandung gespannt wird. Wintrich erklärt sich das Verlorengehen der Regelmässigkeit der Schallwellen daraus, dass die schwingenden Wandungen von ungleich dichten Medien umgeben werden, innen comprimirte Luft, von aussen der gewöhnliche Atmo- 
sphärendruck. Es soll dasselbe eintreten, was sich zeigt, wenn man den Ausschlag eines Pendels an der einen Seite durch einen Widerstand hemmt. Die Schwingungen werden ungleich, unregelmässig und hören bald auf.

Ganz so verhält sich die Sache nun eben nicht. Die Spannung der comprimirten Luft und die Elasticität der gespannten Membran halten einander selbstverständlich in jeder Lage das Gleichgewicht, die gespannte Membran drückt um eben so viel nach innen; als die comprimirte Luft nach aussen drilckt. Von vorn herein sollte man daher erwarten, dass diese beiden Kräfte, welche einander das Gleichgewicht halten und in entgegengesetzter Richtung wirken, sich gegenseitig aufheben müssten und in Folge dessen auch keinen störenden Effect auf die Regelmässigkeit der Schallwellen hervorbringen könnten. Betrachten wir zunächst den Einfluss der gespannten Wandung auf die Intensität und Regelmåssigkeit der Schallwellen. Zunächst ist hierbei zu berücksichtigen, dass die Elasticität organischer Körper nicht direct proportional ist den spannenden Kräften, sondern mit der zunehmenden Spannung der Membran nimmt die Ausdehnbarkeit derselben rasch ab, oder was gleiches bedeutet, der Elasticitätscoëfficient rasch zu. Ist die Membran geradlinig und in einer Ebene gespannt, so bleibt sich der Widerstand, den sie der Wellenbewegung entgegensetzt, nach beiden Seiten gleich und es muss demnach mehr die Intensität als die Regelmässigkeit der Schallwellen abnehmen. Hierin mag auch der Grund liegen, warum, wie Savart nachgewiesen hat, Membranen bei mässiger Spannung besser zu tönenden Schwingungen geeignet sind als bei sehr starker Spannung. Ist aber die Form der Membran sphärisch, oder was in Bezug auf den Effect gleichgültig ist, excentrisch, so wird die Regelmässigkeit der Schallschwingungen dadurch gestört, dass jede nach aussen (d. h. nach der convexen Oberflache der Membran) gerichtete Wellenbewegung die Spannung der Membran zu vermehren sucht, und daher an der Elasticität derselben ein rasch und ungleichmässig wachsendes Hinderniss findet, während alle nach innen (d. h. nach der concaven Fläche der Membran) gerichteten Schwingungen eine ebenfalls rasch und unregelmässig abnehmende Unterstuitzung erhalten. 
Unter diesen Umständen muss demnach nicht blos die Intensität, sondern auch die Regelmässigkeit der Wellenbewegung leiden.

Die Differenz des inneren und äusseren Luftdruckes scheint weniger von Belang zu sein, denn erstens ist nicht abzusehen, warum regelmässige Schallwellen in comprimirter Luft nicht eben so gut zu Stande kommen sollten als in verdünnter, und ferner finden sowohl die Verdünnungs-als Verdichtungswellen der enthaltenen Luft dasselbe und in durchaus gleichmässiger Weise wirkende Hinderniss, denn die Spannung der Luft ist innen direct proportional den drückenden Kräften. Auch Geigel*) schliesst aus seinen Experimenten, dass es nicht die Differenz des Luftdruckes, sondern die Form der gespannten Membran ist, welche das tympanitische Timbre des Percussionsschalles vernichtet.

Dass aber der nicht tympanitische Schall wirklich unter Umständen bei ganz gleicher Spannung der inneren und äusseren Luft zu Stande kommt, davon kann man sich durch die folgenden Experimente leicht überzeugen.

Verschliesst man die eine Oeffnung eines Cylinders durch eine gespannte Membran, so bekommt man bei der mittelbaren Percussion derselben den Schall des Luftraumes im Cylinder; senkt man das untere offene Ende des Cylinders allmälig unter einem schiefen Winkel in Wosser ein, so wird der Schall immer tiefer, bleibt aber tympanitisch, bis der Cylinder so tief eingesenkt ist, dass das Wasser die untere Oeffnung ganz verschliesst. Der Schall wird dann plötzlich nicht tympanitisch.

Ich glaubte nun zunächst die Frage beantworten zu müssen: Welchen Einfluss auf den nichtlympanitischen Schall hat die Höhe des percutirten Luftraumes? Ich bohrte deshalb in die über die obere Oeffnung gespannte Membran ein kleines Loch von 1-2 Mm. Durchmesser. Der durch die mittelbare Percussion der gespannten Membran erbaltene Schall blieb beim Einsenken der unteren Oeffnung des Cylinders in Wasser nichttympanitisch; der Cylinder lässt sich beliebig tief einsenken, ohne dass

*) Geigel, Ueber die physikalische Begründung der Percussionsresultate. Deutsche Klinik 1856. No. 3 . 
eine Compression der enthaltenen Luft entsteht, da dieselbe durch die kleine Oeffnung entweichen kann; das Wasser innerhalb und ausserhalb des Cylinders steht in gleichem Niveau, der Luftraum lässt sich beliebig gross oder klein machen, immer aber erhält man bei der mittelbaren Percussion der Membran denselben nichttympanitischen Schall. Der nichttympanitische Schall zeigt sich also völlig unabhängig von der Grösse des eingescblossenen Luftraums.

Der nichttympanitische Schall ändert sich aber augenblicklich, sobald man durch stärkeres oder schwächeres Andrücken des Plessimeters die Spannung der Membran ändert.

Der nichttympanitische Schall entsteht also nicht in dem eingeschlossenen Luftrame, sondern in der percutirten Membran.

Bindet man über die obere Oeffnung eines unten geschlossenen Cylinders eine ganz sehlaffe Blase, welche den Cylinder wie eine Mütze bedeckt und auf welche man das Plessimeter aufgeklebt hat, so erhält man den tympanitischen Schall des Luftraumes; verschliesst man dagegen denselben Cylinder durch eine gespannte Membran, ohne irgend welche Compression der enthaltenen Luft, so erhält man bei der mittelbaren Percussion einen nicht tympanitischen Schall, dessen Höhe sich nach der Spannung und Grösse der Blase richtet. Erschüttert man dagegen den Boden des Cylinders durch unmittelbare Percussion, so pflanzt sich diese Erschütterung bis auf die Membran fort und man hört den tympanitischen Ton derselben. Die Höhe dieses tympanitischen Tones ist abhängig von der Spannung der Membran; er wird tiefer, wenn man die Membran durchfeuchtet und dadurch die Spannung derselben vermindert; höher, wenn man die Fingerspitze auf die Membran aufsetzt und sie dadurch nöthigt in kleineren Unterabtheilungen zu schwingen. Der Schall wird aber sofort nichttympanitisch, wenn man die Schwingungen der Membran in grösserer Ausdehnung hemmt, z. B. dadurch, dass man einen Finger quer über dieselbe weg legt. Auch dieser nichttympanitische Schall, den man bei der Percussion der Bodenwand des Cylinders erhält, entsteht in der Membran, denn seine Höhe richtet sich nach der 
Spannung derselben, z. B. nach dem Grade des Druckes, den man mit dem Finger auf dieselbe ausïbt.

Spannt man Blase über beide Oeffnungen des Cylinders, so hört man bei der mittelbaren Percussion der einen den tympanitischen Ton der anderen; macht man die. Spannurig der beiden Blasen ungleich, so bekommt man bald einen höheren, bald einen tieferen Ton, je nachdem man die eine oder die andere Blase percutirt, bei ganz gleich bleibender Höhe der Luftsäule des Cylinders.

Es ergiebt sich demnach, dass wir den Schall eines durch stark gespante schallreflexionsfähige Wände allseits geschlossenen Luftraumes durch Percussion uberhaupt nicht erhalten können; wir erhalten immer nur den Schall der Wandungen, und dieser ist tympanitisch, wenn dieselben regelmässige Schwingungen machen können, nichttympanitisch, wenn die Regelmässigkeit der Schwingungen gestört wird, wie es bei der mittelbaren Percussion nothwendig geschehen muss. Die Schallschwingungen des eingeschlossenen Luftraumes werden wahrscheinlich von den Wänden allseits reflectirt, und die geringe Zahl derer, welche die Wände durchdringen, werden durch den Uebergang aus Luft in einen festen Körper und wieder in Luft so geschwächt, dass sie zuletzt unbörbar werden.

Ganz in Uebereinstimmung mit der Entstehung des Schalles in der Wandung finden wir, dass alle die Umstände, welche die Schwingbarkeit der Wandung vermindern, auch den Schall derselben bis auf eine gewisse Entfernung hin dämpfen. Den Beweis dafür liefert folgendes leicht anzustellende Experiment. Man bläst eine Schweinsblase so stark auf, dass sie einen nichttympanitischen Schall giebt. Hemmt man nun die Schwingungen der Blase an irgend einer Stelle durch den Contact eines nicht schwingbaren Körpers, z. B. durch ein mit feuchtem Sande gefültes Glas, welches man mit der Oeffnung nach unten, so dass der Sand auf der Blase lastet, aufsetzt, so findet man, dass der Percussionsschall der Blase immer gedämpfter wird, je mebr man sich dem Rande des Glases nähert.

Indem wir also die Ueberzeugung aussprechen, dass ein be- 
grenzter Luftraum bei der Percussion niemals einen nichttympanitischen Schall giebt, und dass der nichttympanitische Schall immer den Wandungen desselben angehört, müssen wir bemerken, dass der allmälige Uebergang des tympanitischen Schalles in den nichttympanitischen hiermit nicht in Widerspruch steht. Wir beobachten diesen allmäligen Uebergang nur dann, wenn neben dem tympanitischen Schalle des Luftraumes bei allmälig zunehmender Spannung der Wandungen unregelmässige Schwingungen der letzteren nach und nach hörbar werden. Machen wir den in der Luft entstehenden tympanitischen Sehall plötzlich unhörbar, so springt der tympanitische Schall, ohne jenen allmäligen Uebergang, plötzlich in einen nichttympanitischen über.

III.

Der Percussionsschall des Thorax.

Der Thorax giebt bekanntlich bei gesunden Lungen einen nicht tympanitischen Schall. Wie ist derselbe zu erklären? Man darf sich nicht dabei beruhigen, dass eine Lunge, welche wir bis zu dem Volumen, welches sie im Thorax einnimmt, aufblasen, eben auch einen nichttympanitischen Schall giebt; eine aufgeblasene Lunge enthält immer comprimirte Luft, die Lunge ist im Thorax aber eben nicht aufgeblasen, denn die in ihr enthaltene Luft ist für gewöhnlich nicht comprimirt. Die im Thorax enthaltene gesunde Lunge unterscheidet sich nur durch ihre stärkere elastische Anspannung und die dadurch bewirkte Vergrösserung ihres Volums und ihres Luftgehaltes von einer contrahirten Lunge, und doch scheint auf den ersten Blick in keinem dieser Verhältnisse die Ursache des nichttympanitischen Schalles zu liegen, da, wie Wintrich gezeigt hat, durch Dehnung und Anspannung einer Lunge ihr tympanitischer Schall nicht verloren geht.

Wintrich sucht über diese Schwierigkeiten durch folgendes Experiment hinweg zu kommen: „Man binde eine sehr elastische gesunde Lunge mittelst der Trachea an ein Rohr, welches oben in eine Glasglocke luftdicht eingekittet ist und ausserhalb der Glocke einen ebenfalls luftdicht schliessenden Hahn besitzt, und setze 
dann bei offenem Hahn die Glocke auf den Teller der Luftpumpe, welche man allmälig wirken lässt. Wird die Luft in der Glocke auch nur um $\frac{1}{10} \overline{0}$ Atmosphärendruck vermindert, so drückt die äussere Luft schon so stark auf das contractile Gewebe in den Lufträumen der Lunge, dass diese aufgebläht wird etwa wie bei einer Inspiration. Schliesst man den Hahn und hebt die Glocke $a b$, so giebt die aufgeblähte Lunge einen nichttympanitischen Schall *)." In wiefern sich eine durch diese absonderlich künstliche Methode aufgeblasene Lunge von einer ganz einfach aufgeblasenen Lunge unterscheiden soll, ist durchaus nicht abzusehen; es ist gerade so, als wenn man vor Eröffnung des Thorax die Trachea luftdicht verschliessen würde; auch in diesem Falle würde die Lunge nach Eröffnung des Thorax vermöge ihrer Elasticităt die in ihr enthaltene Luft comprimiren, während doch die im uneröffneten Thorax enthaltene Lunge für gewöhnlich keine comprimirte Luft enthält. Eben so wenig kann mañ bei der im Thorax enthaltenen Lunge von einer „Spannung des Gewebes mit ungleicher Dichtigkeit der umgebenden Medien" reden; denn so weit das Lungengewebe überhaupt von Luft umgeben ist, steht es auch überall unter demselben Luftdrucke. Die Druckdifferenz, von der Wintrich so viel redet, betrifft nur die Thoraxwand; diese steht allerdings, wie Donders gezeigt hat, aussen unter dem Drucke der Atmosphäre, innen unter demselben Drucke minus der Lungenelasticität; aber bevor man den nichttympanitischen Schall des Thorax davon ableitet, sollte man billiger Weise erst bewiesen haben, dass die Thoraxwand überhaupt beim Percussionsschall eine active Rolle spielt. Unserer Ueberzeugung nach giebt die im Thorax aufgespannte Lunge auch aḅgesehen von etwanigen stehenden Schwingungen der Thoraxwand einen nichttympanitischen Schall, und man darf sich dadurch nicht irre machen lassen, dass der tympanitische Schall einer, aus dem Thorax herausgenommenen Lunge bei geradliniger Anspannung derselben in einer Ebene nicht verloren geht. Eine geradlinig in einer Ebene gespannte Membran giebt auch einen tympanitischen Schall, weil sie nach beiden Seiten hin mit gleicher Leichtigkeit schwingen kann; bei excentrischer Span*) l. c. S. 30 . 
nung*), wie es bei einer durch Aufblasen stark gespannten Blase der Fall ist, wird der Schall, wie wir gesehen haben, deshalb nichttympanitisch, weil alle nach aussen gerichteten Schwingungen derselben an der Elasticität der Membran ein rasch und ungleichmässig wachsendes Hinderniss finden; gerade so verhält sich aber die excentrisch aufgespannte Lunge. Beiläufig bemerke ich nur, dass es nicht etwa blos die Pleura pulmonalis ist, deren excentrische Spannung den nichttympanitischen Schall bestimmt; man kann sich davon durch das Abpräpariren der Pleura einer aufgeblasenen Lunge überzeugen, was für kleinere Strecken wenigstens sehr leicht gelingt.

Wir haben also gesehen, dass eine begrenzte Luftmasse bei der Percussion immer einen tympanitischen Sehall giebt; dass ferner der vichttympanitische Schall sich in allen seinen Qualitäten abhängig zeigt von dem Verhalten der Wandungen des percutirten Luftraumes, unabbängig dagegen von der Menge der enthaltenen Luft; und dass endlich ein von gespannten schallreflexionsfähigen und zu tönenden Schwingungen geeigneten Wänden allseits eingeschlossener Luftraum bei der Percussion überhaupt keinen (wenigstens auf die gewöhnliche Distanz) wabrnebmbaren Schall giebt, sondern dass wir unter diesen Umständen allemal den Schall der Wandungen bekommen. Alle diese Sätze finden Anwendung bei der Percussion der Lungen. Wir haben schon bei Gelegenheit des tympanitischen Schalles einfach retrahirter Lungen gesehen, dass sich die in der Luft der Lungen entstandenen Schallwellen durch die Bronchien und die Trachea nicht nach aussen fortpflanzen, sondern wahrscheinlich schon in den feineren Bronchien zu Grunde gehen, denn sonst müsste der tympanitische Schall einer einfach retrahirten Lunge beim Verschluss der Trachea tiefer werden. Ebenso verhält sich aber die Sache bei der im Thorax enthaltenen Lunge, und wir müssen daher behaupten, dass die im Thorax excentrisch ausgespannte Lunge sich bei der Percussion wie ein von schallreflexionsfähigen und zu tönenden Schwingungen geeigneten Wän-

*) Excentrisch gespannt nenne ich der Kürze wegen einen Körper, der vermöge seiner Spannung das Bestreben hat, sich nach allen seinen Dimensionen, also concentrisch, za verkleinern. 


\section{6}

den eingeschlossener luftraum verhalte, wir müssen den nichttympanitischen Percussionsschall derselben von ihrer excentrischen Spannung ableiten, und müssen daher endlich die Quelle des nichttympanitischen Schalles der im Thorax enthaltenen Lunge im elastischen Lungengewebe selbst suchen, gerade so gut wie wir den nicbttympanitischen Schall überhaupt nie in einem begrenzten Luftraum, sondern immer nur in den elastischen Wandungen desselben zu Stande kommen sahen.

Die Betheiligung der Thoraxwandungen am Percussionsschalle der Brust anlangend, so kann die Frage nur die sein, ob die Thoraxwand an sich den nöthigen Grad von Spannung besitze, um durch Percussion in tönende Schwingungen versetzt zu werden. Man sieht ein, dass man diese Frage nicht von vorn herein für entschieden ansehen darf, wie es Hoppe thut, wenn er die $\mathrm{Ge}-$ setze, welche für die Schwingungen ebener und gebogener Platten gelten, auf die Percussionserscheinungen des Thorax zu übertragen sich bemüht. Eine durchaus befriedigende Beantwortung dieser Frage ist fast unmöglich, denn percutirt man nach Entfernung sämmtlicher Brusteingeweide den Thorax, so bekommt man, wie dies Wintrich gegen Mazonn geltend macht, nicht den Schall der Thoraxwandung, sondern den des eingeschlossenen Luftraumes, während grössere aus der Thoraxwandung herausgeschnittene Stücke, eben weil sie herausgeschnitten sind, nicbt mebr die spannung besitzen, welche dem unverletzten Thorax zukommt; wenn nun Mazonn bei der Percussion solcher Stucke, welche er in einen Schraubstock einspannte, dennoch einen Schall erhielt, welcher zwar nicht laut, aber durchaus auch nicht dem Schenkelschalle gleich war, so spricht dies allerdings dafür, dass um so mehr den unverletzten Thoraxwänden die Fähigkeit tönender Schwingungen zugeschrieben werden darf. Skoda*) giebt das Factum zu, macht aber dagegen geltend, ,der so erhaltene Schall sei nicht der Schall der Brustwand allein, sondern zugleich ein Schall der umgebenden Luft, und dieser Schall der umgebenden Luft sei nicht eine Verstärkung des Schalles der Brustwand, sondern ein selbständiger Schall" - gegen diese Einwürfe Skoda's ist zu erwidern, dass

*) S. 33. 
nur begrenzte Lufträume selbständig schallen können, dass im vorliegenden Falle die Luft also nur als Schallleiter dient, wie sie es eben bei der Percussion so gut wie bei fast allen Tönen, die wir hören, thun muss.

Beweisender ist das gleichfalls von Mazonn (p. 3.) zuerst vorgebrachte Factum, dass man den Percussionsschall des Thorax auch dadurch dämpfen kann, dass man eine Hand fest auf die eine Seite der Brust eines gesunden Menschen andrücken lässt; man muss aber, um diese geringe Dämpfung wahrzunehmen, unmittelbar am Rande der aufgelegten Hand percutiren. Wir glauben es demnach für ausgemacht halten z11 dürfen, dass die Thoraxwandungen durch Percussion in tönende Schwingungen versetzt werden können, und der so erhaltene Schall muss immer ein nichttympanitischer sein, weil wegen des elastischen concentrischen Zuges der Lungen alle nach aussen gerichteten Schwingungen der Thoraxwand einen pasch und ungleichmässig wachsenden Widerstand finden. Daraus folgt aber nicht, dass, wie Hoppe annimmt, der Percussionsschall des Thorax unter allen Umständen und nur durch Transversalschwingungen der Wandungen zu Stande käme; wenn z. B. nach Wintrich's Aussage der tympanitische Schall über grossen Cavernen beim Verscbluss der Nasen- und Mundöffnung tiefer wird, so folgt schon daraus, dass der Schall nicht in der Thoraxwandung, sondern im Luftraume der Caverne selbst entstanden ist.

Auch der tympanitische Schall, den wir über lufthaltigem Lungengewebe beim Nachlass der elastischen Spannung desselben in Folge einer Durchtränkung mit Exsudaten und bei Retraction der Lunge in Folge reichlicher pleuritischer Ergüsse erhalten, entsteht meiner Ansicht nach nicht durch Schwingungen der Thoraxwandungen, sondern in der Luft der Lunge selbst, welche unter diesen Umständen in Folge des Nachlassens der excentrischen Spannung der Lunge im Stande ist einen tympanitischen Schall zu geben. Die Thoraxwandung spielt unter diesen Verbältnissen eine rein passive Rolle, und es kommen in der That, wie dies auch Hoppe hervorhebt, in diesen Fällen alle Umstände zusammen, um die Spannung der Thoraxwandung zu vermindern. Uebereinstimmend damit finden wir, dass in allen diesen Fällen der tympanitische 
Percussionsschall um so deutlicher ist, je nachgiebiger, d. h. je weniger zu selbsttönenden Schwingungen geeignet, die Thoraxwandung ist. Bleibt bei dem erörterten pathologischen Verhalten der Lungen die Thoraxwandung dennoch hinlänglich gespannt und $z u$ tönenden Schwingungen geeignet, so verhält sie sich ähnlich wie eine straff angespannte Blase, mit welcher wir diẻ Oeffnung eines Gefässes verschlossen haben; selbst wenn eine solche Blase mehrere Zoll gross ist, so erhalten wir bei der mittelbaren Percussion derselben doch keinen tympanitischen Schall. Gerade daraus also, dass der Percussionsschall unter den erwähnten Umständen tympanitisch ist, schliessen wir, dass er nicht in der Thoraxwandung entstehe.

Für diejenigen Fälle jedoch, bei welchen wir eine Betheiligung der Thoraxwandungen annehmen, also für den nichttympanitischen Percussionsschall, müssen wir auch den Einfluss berücksichtigen, welchen feste, luftleere, der Thoraxwandung anlagernde Körper auf den Percussionsschall ausüben; durch dieselben wird nämlich der Schall nicht blos an der Berührungsstelle, sondern in weiterem Umfange gedämpft, so dass man also in der Nähe luftleerer Organe, welche der Thoraxwand mit einem gewissen Drucke anliegen, auch über der angrenzenden normalen lufthaltigen Lunge einen gedämpften Schall erhält; es ist wabrscheinlich, dass diese Dämpfung um so ausgebreiteter ist, je stärker der Druck, den diese Organe auf die Thoraxwand ausüben, wie es Mazonn (S. 22.) bei Vergrösserung der Leber beobachtet hat. Das Factum war längst bekannt, aber missverstanden und falsch gedeutet worden. Indemman nämlich den Percussionsschall der Brust unmittelbar auf die in ibir enthaltenen Organe bezog, glaubte man durch , oberflächliches Percutiren" die unmittelbar unter der Brustwand, durch „tiefes Percutiren” die tiefer liegenden Organe erforschen zu können. Schon Piorry*) spricht sich in diesem Sinne aus: "Durch ein schwächeres Percutiren lernt man die oberflächlichen Lungenschichten kennen; wird es allmälig stärker, so lernt man die Dichtigkeit der Lungen in der Tiefe kennen."

*) Cf. Mailliat, Traité pratique de Percussion. 1843. p. 75. 


\section{9}

Noch entschiedener vertritt $\mathrm{Hughes}$ ) dieselbe Meinung: „Bei der Untersuchung, ob ein Ton aus der Tiefe oder von der Oberfläche kommt, muss man den Anschlag der Finger in verschiedener Stärke und wechselnd ausfïhren. Liegt zwjschen einem soliden Gebilde und den Brustwänden eine dünne Lage gesunder Lunge, dann erhält man durch leichtes und zartes Klopfen jenen Ton, welcher dieser Lage entspricht, während man beim festen Andrücken und starken Anschlagen den dumpfen Ton vernimmt, welcher den tiefer liegenden soliden Gebilden entspricht."

Von derselben Ansicht ging endlich auch Conradi**) aus, indem er den dumpfen Schall, den man über luftleeren compacten Organen durch die Percussion erhält, als Leerheit (Leberleerheit, Herzleerheit u. s. w.) bezeichnete und davon den gedämpften Schall, den man bei der Percussion der angrenzenden Thoraxwandung erhält, als Dämpfung (Leberdämpfung, Herzdämpfung u. s. w.) unterschied. Auch Conradi giebt ausdrücklich an, dass man sich, um die Grenzen der „Dämpfung” zu finden, einer starken Percussion bedienen müsse. Z. B. S. 14: „Um den die Herzdämpfung umgebenden Raum zu bestimmen, ist eine kräftige Percussion nothwendig, weil nur starke Schwingungen der Brustwand die lufthaltige Lungensubstanz durchdringen und zu dem unter ihr vergrabenen Herzen dringen können.” "Um den Theil des Herzens zu bestimmen, der frei und nackt der Brustwand anliegt, darf man nur leicht mit dem Finger auf den Plessimeter klopfen, da auch geringe Vibrationen der Brustwand mit Leichtigkeit zu der direct anliegenden Herzsubstanz gelangen können, starke aber sich leicht den lufthaltigen Nachbarorganen mittheilen und so einen unbestimmten Ton hervorbringen können."

Was man sich in diesem Falle unter einem "unbestimmten Ton" zu denken habe, wird nicht angegeben; durch den Versuch kann man sich leicht uberzeugen, dass der Percussionsschall der Herzgegend bei starker Percussion intensiver ist als bei schwacher

") Hughes, Pratice of Auscultation and other modes of physical diagnosis in diseases of the Lungs and Heart. London 1854.

*) Conradi, Ueber die Lage und Grösse der Brustorgane, der Leber und Milz. Giessen 1848.

Archiv f. pathol. Anat. Bd, XI. Hfr. 2. 
Percussion, wie es eben überall am Thorax geschieht. Dass man aber, wie es Hughes ausdrücklich angiebt und auch Conradi anzunehmen scheint, an einer und derselben Stelle bei schwacher Percussion einen intensiveren Schall als bei starkem Anscblag erhalten könne, ist eine Annahme, welche allen physikalischen Gesetzen so sehr widerspricht, dass wir uns füglich wohl eine ausfuhrliche Widerlegung sparen können. Die Thatsache aber, dass in der Umgebung luftleerer Organe, welehe die Schwingungen der Thoraxwand in einer gewissen Ausdehnung gänzlich hemmen, der Schall weniger intensiy ist als da, wo sich die Percussionserschilterung ungehindert nach allen Seiten ausbreiten kann, lässt sich leicht aus der anderweitig nachgewiesenen Betheiligung der Thoraxwandung am Percussionsschall erklären, und berechtigt keineswegs zu dem Schlusse, dass man durch die Percussion des Thorax mebr als die Schwingungstähigkeit der Thoraxwandung selbst und der ihr unmittelbar anlagernden Organe erfahren könne. Die Unterscheidung zwischen Schall-Dämpfung und Leerheit im Sinne Conradi's erfährt also eine gewisse Einschränkung. Wenn in der Umgegend einer auf den Thorax angedrückten Hand der Sehall gedämpft ist, so wird Niemand zwischen Handdämpfung und Handleerheit unterscheiden wollen. Wie gross die erwähnte Einschränkung in jedem einzelnen falle sein muss, das hängt von der Schwingungsfähigkeit der T'horaxwandungen $a b$, die bei verschiedenen Individuen eine verschieden grosse zu sein scheint.

IV.

Skoda's voller und leerer Percussionsschall.

Ich habe es bisher vermieden, zur Bezeichnung verschiedener Schallqualitäten einen anderen Ausdruck als den des tympanitischen und nichttympanitischen Timbres und die Schallintensität (resp. Dämpfung) zu benutzen. Was nämlich die Unterschiede in der Schallhöhe anlangt, so haben dieselben, wie Skoda angiebt, den geringsten praktischen Werth, da sie nicht von einer einzigen, sondern von mehreren Bedingungen abhängen, die sich unserer Beobachtung entziehen, z. R. beim nichttympanitischen Schalle von 
der Spannuńg der Wandungen, beim tympanitischen Schalle von Cavernen, von der Weite der Communication mit der Trachea u. s. w. Man kann demnach die Reihe rom hohen zum tiefen Schall durchaus nicht, wie Mazonn und Traube*) thun, für gleichbedeutend mit der Reihe vom vollen zum leeren Schall annehmen. Denn sucht man einmal nach einer Ejgenschaft des Schalles, aus welcher man auf die Grösse des sehallenden Körpers schliessen kann, so sagt Skoda mit vollem Rechte: ,auch nach der Schallhöhe schliessen wir nicht auf die Grösse des schallenden Körpers." Dass man nun trotz alles Suchens eine solche Eigenschaft des Schalles nicht gefunden hat, und dass auch Skoda nicht im Stande ist, das, was er voll und leer nennt, genau zu definiren, liegt ganz einfach darin, dass es überhaupt gar keine Schallqualität giebt, welche in directer Abhängigkeit von der Grösse des schallenden Körpers stände. Es ist schon bezeichnend genug, dass im Sprachgebrauch für eine solche Eigenschaft des Schalles gar kein Wort existirt; die Ausdrücke voll, volltönend, sonor, deren sich Skoda bedient, werden in ganz anderem Sinne genommen, kein Mensch wird aus der Sonorität der Stimme auf die Grösse der Stimmbänder schliessen wollen, oder etwa behaupten, dass eine grosse Geige einen volleren Ton haben müsse als eine kleine. Versuchen wir es uiberhaupt jemals einen derartigen Schluss zu machen, so sind die Eigenschaften des Schalles, auf die wir unser Urtheil gründen, keine anderen als die Schallhöhe und die Intensität, und sind wir auch hierbei vielfachen Tåuschungen unterworfen. Wir wissen z. B. aus Erfahrung, dass der Ton einer grossen Orgelpfeife tiefer ist als der einer kleinen, und sind daher geneigt, einen Ton einer solchen Pfeife, der um eine Octave tiefer ist als der einer anderen, auf einen grösseren schallenden Körper zu beziehen; und doch können die Pfeifen durchaus gleich gross sein, wenn die eine oben offen, die andere aber verschlossen ist. Vergleichen wir, wie es Körner angiebt, den Percussionston zweier cylindrischer Luftsäulen von gleicher Höhe, aber auffallend verschiedenem Durchmesser, so wird es allerdings Niemandem einfallen, den über dem engeren Cylinder hör-

*) Ueber den Zusammenlıang der Herz- und Nierenkrankheiten S. 47. 
baren Ton in den weiteren zu verlegen, und umgekehrt; es ist aber lediglich die Intensität des Schalles, welche unser Urtheil bestimmt.

Von anderen Seiten glaubte man die Reihe Skoda's vom vollen zum leeren Schall auf die Oscillationsdauer des tönenden Körpers zurückführen zu können. Man hat sich dabei an die Aeusserung $S k$ oda's gehalten, dass der volle Schall länger anhaltend und wie über einen grösseren Raum verbreitet erscheine als der leere; aus diesem Grunde identificirte Mazonn (1. c. S. 40.) die Reihe vom hohen zum tiefen Schall mit der vom vollen zum leeren, da allerdings die Schwingungsdauer tiefer Töne länger ist als die hoher Töne; die. Einwendungen Wintrich's (1. c. S. 41.) widerlegen Mazonn nicht, denn Mazonn spricht ausdrücklich von gleichmässig gespannten Saiten, während Wintrich's Experimente eine ungleiche Anspannung derselben voraussetzen. Eben so wenig wird der Werth des Factums; dass tiefe Töne fur gewöhnlich eine längere Oscillationsdauer laaben als hohe, dadurch vermindert, dass Geigel*) bei dieser Gelegenheit darauf aufmerksam macht, dass hohe sowohl als tiefc Töne dieselbe Fortpflanzungsgeschwindigkeit haben, und wenn Geigel fortfăhrt, dass es sich ,hier uberdies nur un die Fähigkeit tönender oder schallender Körper handle, die in ihnen erzeugten Vibrationen längere oder kürzere Zeit, je nach ihrem Elasticitätsgrade, zu conserviren" so hat dies mit der Grösse des schallenden Körpers, um die es in Skoda's Reihe vom vollen zum leeren Schall sich nun einmal ausschliesslich handelt, durchaus nichts zu thun.

In der funften Auflage (S. 34.) seiner Abhandlung hat Skoda selbst die Verwerthung der längeren Oscillationsdauer für diese Frage wesentlich beschränkt, indem er angiebt, dass der volle sowohl als der leere Ton eines Instrumentes lang oder kurz sein könne, und dass das beim Percutiren erregte metallische Klingen in der Regel bédeutend länger, aber dennoch leerer sei, als der begleitende tympanitische oder nichttympanitische Schall. Gleichzeitig aber wirft Skoda zum Beweise seiner Bebauptung, dass es

") Ueber die physikalische Begründung der Percussionsresultate. Deutsche Klinik 18j6. S. 27. 
eine Eigenschaft des Schalles gebe, aus der wir auf die Grösse des schallenden Körpers schliessen, die imponirende Frage auf: „Lässt sich das Unisono pianissimo mehrerer Violinen durch das Forte einer Violine supfliren?" Die Antwort kann nicht zweifelhaft sein, aber die Gründe für diese musikalische Unmöglichkeit sind so mannigfaltig und die hiehergehörigen akustischen Beziehungen so verwickelt, dass man meiner Ueberzeugung nach einen so einfachen Schluss, wie es Skoda gethan hat, aus so complieirten Verhältnissen nicht ziehen darf. Ich will nur daran erinnern, dass streng genommen ein Unisono ein musikalisches Desiderat ist. Abgesehen davon, dass schon sehr geringe Differenzen in der Schallhöhe und im T'empo sich bemerklich machen, die sich auch bei der grössten Virtuosität einer Capelle kaum ganz dürften vermeiden lassen, so hat auch fast jedes einzelne Instrument sein eigenes Timbre; ferner haben wir eine Empfindung dafür, in welcher Richtung wir einen Ton hören, und es macht demnach einen verschiedenen Eindruck, ob wir einen Ton nur von einem Punkte aus hören oder gleichzeitig von vielen Punkten aus, wie es beim Unisono mehrerer Instrumente eben nothwendig der Fall sein muss. Suchen wir aber in dem von Skoda gewählten Beispiele nur die nächsten Bedingungen auf, so finden wir in dem Gegensatze des Pianissimo und Forte zunächst Verhältnisse, welche von der Oscillations-Amplitide jeder einzelnen Schallwelle abhängig sind; in Betracht kommt ferner die Menge der gleichzeitig gehörten identischen Schallwellen, die natürich bei dem Unisono pianissimo mehrerer Violinen beträchtlicher ist als bei dem Forte nur einer Violine. Aus der Oscillations-Amplituide können wir nicht auf die Grösse des schallenden Körpers schliessen, denn auf einem und demselben Instrumente kann man jeden Ton ganz nach Belieben mit grosser oder geringerer Oscillations-Amplitüde hervorbringen. Die Frage kann also nur die sein: „Kann man aus der Menge der unser Ohr gleichzeitig treffenden identischen Schallwellen auf die Grösse des schallenden Körpers schliessen?" Experimentell stellt sich die Frage so: ,Welche Differenzen in der Wahrnehmung des Schalles treten ein, je nachdem in allen ubrigen Beziehungen identische Schallwellen unser Ohr gleichzeitig entweder in gerin- 
gerer oder grösserer Menge treffen?" Ein sehr günstiges und einfaches Beispiel hierfür liefert uns jede Stimmgabel. Eine Stimmgabel tönt, in freier Luft gehalten, viel schwächer, als wenn man sie auf einen Tisch, auf einen Kasten $u$. s. w. aufsetzt. Der mechanische Vorgang hierbei ist bekanntlich folgender. Eine in freier Luft gehaltene Stimmgabel theilt ihre Schwingungen blos der Luft mit; setzen wir dieselbe auf den Tisch, so theilen sich die Schwingungen nicht blos der Luft, sondern auch dem Tische mit, derselbe theilt sich durch Knotenlinien in verschiedene Unterabtheilungen, welche jede für sich denselben Ton geben als die Stimmgabel; wir haben hier also das vollständigste Unisono. Die Oscillations-Amplitüde der Schwingungen, welche der Tisch macht, ist abhängig von der Intensität der einzelnen Impulse, die ihm von der Stimmgabel mitgetheilt werden. Die OscillationsAmplitilde der Stimmgabel selbst aber richtet sich nach der Kraft des Anschlages, durch den sie in Schwingungen versetzt wurde. Setzen wir die Stimmgabel auf den Tisch; so wirkt dieselbe Kraft auf eine grössere Masse; wir sollten also nach allen mechanischen Grundsätzen eher eine Abnahme der Oscillations-Amplitüde erwarten, jedenfalls aber kann eine Vergrösserung derselben nicht eintreten. Wir haben hier wieder ein Beispiel, wie leicht wir Irrthumern ausgesetzt sind, wenn wir unsere sinnlichen Wahrnehmungen unmittelbar auf die physikalischen Gründe derselben übertragen. Intensität des Tones und Oscillations-Amplituide der Schallwelle sind in der Physik gleichbedeutend; etwas anderes aber ist der physikalische Begriff einer Schallwelle, etwas anderes die sinnliche Wahrnehmung eines Tones. Das Experiment zeigt also, dass bei vollkommen gleicher Oscillations-Amplitude in allen übrigen Beziehungen identischer Schallwellen doch die Intensităt des wahrgenommenen Tones eine grössere ist, wenn die Schallwellen in grösserer Menge gleichzeitig unser ohr treffen, begreiflicher Weise deshalb, weil alle Schwingungen, welche das Trommelfell selbst macht, intensiver sind, wenn es von vielen Schallwellen gleichzeitig, als wenn es von wenigen getroffen wird. Zugleich sehen wir, dass ein und derselbe Ton, je nachdem er (bei gleicher Oscillations-Amplitüde aller einzelnen Schwingungen) von ein em grossen 
oder kleinen Körper ausgeht, nur in derIntensität unserer sinnlichen Wahrnehmung eiñe Verschiedenheit zeigt; dass man aber daraus nicht auf die Grösse des schallenden Körpers schliessen könne, haben wir schon gesehen und wird von Skoda mit Recht hervorgehoben.

\section{Resumé.}

1. Der tympanitische Percussionsschall des Thorax entsteht weder in der Thoraxwand noch im Lungengewebe, sondern immer in der in den Lungen enthaltenen Luft.

2. Der nichttympanitische Schall des Thorax kommt theils jm Lungengewebe, theils in der Thoraxwand selbst zu Stande. Die in der Lunge enthaltene Luft ist dabei nur in so weit betheiligt, als sie die Schwingungen nicht hemmt. Alle Verhältnisse, welche die Schwingungen der Thoraxwandung und des. Lungenparenchyms hemmen, dämpfen den Percussionsschall.

3. Die Unterscheidung zwischen Schall-Dämpfung und Leerheit im Sinne Conradi's erfährt eine gewisse Einschränkung, die gerade so gross ist, wie die Betheiligung der Brustwand am Percussionsschalle.

4. Es giebt keine Schallqualität, welche in directer und alleiniger Abhängigkeit von der Grösse des schallenden Körpers stünde. Die Rejbe Skoda's vom vollen Schall zum leeren ist demnach zu streichen. 